Check for updates

Cite this: Nanoscale Adv., 2019, 1, 367

\title{
In vivo fate of free and encapsulated iron oxide nanoparticles after injection of labelled stem cells $\uparrow$
}

\author{
Sumaira Ashraf, (iD *a Arthur Taylor, ${ }^{\text {bc }}$ Jack Sharkey, ${ }^{\text {bc }}$ Michael Barrow, (D) d \\ Patricia Murray, ${ }^{\text {bc }}$ Bettina Wilm, (D) bc Harish Poptani, ${ }^{\text {bc }}$ Matthew J. Rosseinsky, ${ }^{d}$ \\ Dave J. Adams (D) de and Raphaël Lévy (D) *a
}

\begin{abstract}
Nanoparticle contrast agents are useful tools to label stem cells and monitor the in vivo bio-distribution of labeled cells in pre-clinical models of disease. In this context, understanding the in vivo fate of the particles after injection of labelled cells is important for their eventual clinical use as well as for the interpretation of imaging results. We examined how the formulation of superparamagnetic iron oxide nanoparticles (SPIONs) impacts the labelling efficiency, magnetic characteristics and fate of the particles by comparing individual SPIONs with polyelectrolyte multilayer capsules containing SPIONs. At low labelling concentration, encapsulated SPIONs served as an efficient labelling agent for stem cells. The bio-distribution after intracardiac injection of labelled cells was monitored longitudinally by MRI and as an endpoint by inductively coupled plasma-optical emission spectrometry. The results suggest that, after being released from labelled cells after cell death, both formulations of particles are initially stored in liver and spleen and are not completely cleared from these organs 2 weeks post-injection.
\end{abstract}

Received 23rd July 2018

Accepted 16th September 2018

DOI: $10.1039 / \mathrm{c} 8 \mathrm{na00098k}$

rsc.li/nanoscale-advances
Magnetic resonance imaging (MRI) offers much higher spatial resolution $(50 \mu \mathrm{m})$ than BLI allowing in vivo cell tracking combined with detailed anatomical information at the level of individual organs. ${ }^{16}$ Appropriate MRI contrast agents ${ }^{17,18}$ for cell tracking include superparamagnetic iron oxide nanoparticles (SPIONs) which generate a negative contrast. ${ }^{11,19-24}$ The uptake efficiency of SPIONs by cells is strongly influenced by the functionalization of SPIONs. ${ }^{21,25}$ Recently, positively-charged DEAE (diethylaminoethyl)-dextran coated SPIONs have been synthesized for enhanced cellular uptake and MRI contrast. ${ }^{20,22}$ Upon cell uptake, the clustering and confinement of these particles in endosomal and lysosomal compartments affects their abilities to alter the relaxation rate and hence relaxivity of the surrounding water molecules. ${ }^{26,27}$ The critical parameter for imaging is the contrast obtained after cell labelling rather than the solution relaxivity of the particles. ${ }^{\mathbf{2 0 , 2 2}}$

One potential approach to enhance SPIONs uptake by cells, is to assemble them inside polymeric capsules resulting in the packing of large amount of individual nanoparticles within a confined volume. ${ }^{28-30}$ Polyelectrolyte multilayered (PEM) and multifunctional capsules are fabricated by layer-by-layer (LbL) assembly of oppositely charged polymeric layers around a template. ${ }^{31}$ Their design can be tailored using multiple strategies and particle loading can be enhanced by including additional layers of particles during the capsule's assembly. ${ }^{32}$ The choice of the final polymer layer determines their cellular interaction, uptake efficiency and hence internalization. It has been observed that a final layer of positively charged polymers results in increased uptake of capsules. ${ }^{33}$ For long term cellular

\footnotetext{
Department of Biochemistry, Institute of Integrative Biology (IIB), University of Liverpool, Liverpool, UK. E-mail: rapha@liverpool.ac.uk; sumaira@liverpool.ac.uk ${ }^{b}$ Department of Cellular and Molecular Physiology, Institute of Translational Medicine (ITM), University of Liverpool, Liverpool, UK

${ }^{c}$ Centre for Preclinical Imaging, Institute of Translational Medicine (ITM), University of Liverpool, Liverpool, UK

${ }^{d}$ Department of Chemistry, University of Liverpool, Liverpool, UK

${ }^{e}$ School of Chemistry, University of Glasgow, Glasgow, UK

$\dagger$ Electronic supplementary information (ESI) available. See DOI: 10.1039/c8na00098k
} 
imaging the walls of the capsules can be made of nonbiodegradable materials. ${ }^{34}$

We and others have shown that most imaging contrast labelled cells used for regenerative medicine therapies including stem cells, die after injection. ${ }^{11,15,35}$ Therefore, understanding the in vivo fate of cell-labelling nanoparticles, especially following the death of the labelled cells is important for the interpretation of imaging studies and to assess the risk of toxicity. In the current study, we focus on the effect of formulation on the fate of SPIONs after the in vivo injection of labelled cells. More specifically, we labelled mouse bone marrow derived mesenchymal stem cells (mMSCs) with free and PEM-encapsulated SPIONs. First the solution relaxivities, magnetic properties, toxicity, and cell labelling efficiency of both formulations of particles are compared. Then, after intracardially (IC) injecting the labelled cells in the left ventricle of mice, the animals were longitudinally imaged by BLI and MRI until 14 days post injection. The cellular bio-distribution and viability was monitored by BLI, whilst MRI allowed visualization of the in vivo fate of particles after cell death. At the end point of experiments ( 2 weeks post-injection), the amount of elemental iron inside the organs was measured to analyze the accumulation and elimination of both formulations of SPIONs. Our studies demonstrate a similar accumulation and elimination pattern of particles injected via labelled cells to what has been reported for the particles directly injected in the blood stream. ${ }^{36-40}$

\section{Results and discussion}

Preparation and characterization of free and encapsulated SPIONs

To evaluate the effect of incorporation of the SPIONs into capsules, magnetization curves were acquired for capsules and free particles; no difference was observed between the magnetic properties of the free and encapsulated SPIONs (Fig. 1). Whilst the properties of individual particles were not affected by encapsulation, the large number of particles in a single object resulted in higher magnetic forces that enabled magnetic separation of particles during purification from excess reactants. Thus, during LbL assembly of particles in polymeric capsules, as the number of particles per capsule increased, their magnetic separation by a bar magnet became possible. After deposition of 3 layers of SPIONs, for further layer deposition, the excess reactants were therefore removed by magnetic separation instead of centrifugation. The transmission electron micrographs (Fig. 1a and b) show that particles did not fuse inside the capsules; they retained their identity as individual particles (as indicated by the magnetization curve; Fig. 1c). The crystalline structure and particle size $(\sim 8 \mathrm{~nm})$ was confirmed by powder XRD (pXRD) before encapsulation (Fig. SI.1a $\uparrow$ ). Their hydrodynamic diameter in water was measured by dynamic light scattering (DLS, $\sim 85 \mathrm{~nm}$, Fig. SI.1b†). The hydrodynamic diameter of capsules cannot be measured accurately by DLS because of their dimension ( $\mu \mathrm{m}$ size) and sedimentation behavior.

\section{Effect of encapsulation of particles on the solution relaxivity}

To determine the effect of encapsulation on the solution relaxivity, the water relaxation rates of particles at different concentrations (based on Fe content) were measured by MRI and plotted as function of concentration (Fig. 2). Encapsulation of SPIONs resulted in a drop in solution relaxivity due to hindered access to the solution water protons and is similar to the previous findings of the entrapment of free particles inside the lysosomes..$^{\mathbf{2 0}, 22,32}$ We used non-biodegradable polymers to encapsulate the SPIONs to avoid the changes in solution relaxivity and the resultant image contrast due to disassembly of polymeric capsules. ${ }^{34}$

\section{Cell labelling efficiency and toxicity of SPIONs}

mMSCs were chosen for this study because they are similar to human bone marrow derived hMSCs which are being used in clinical trials, ${ }^{\mathbf{4 1 , 4 2}}$ and it has already been shown that these cells uptake free SPIONs and other nanoparticle probes with good efficacy. ${ }^{12,22}$ To evaluate the cell labelling efficiency and toxicity of free and encapsulated SPIONs, cells were labelled with suspensions of particles (encapsulated or free) having comparable Fe content. The cells were imaged by MRI to quantify the
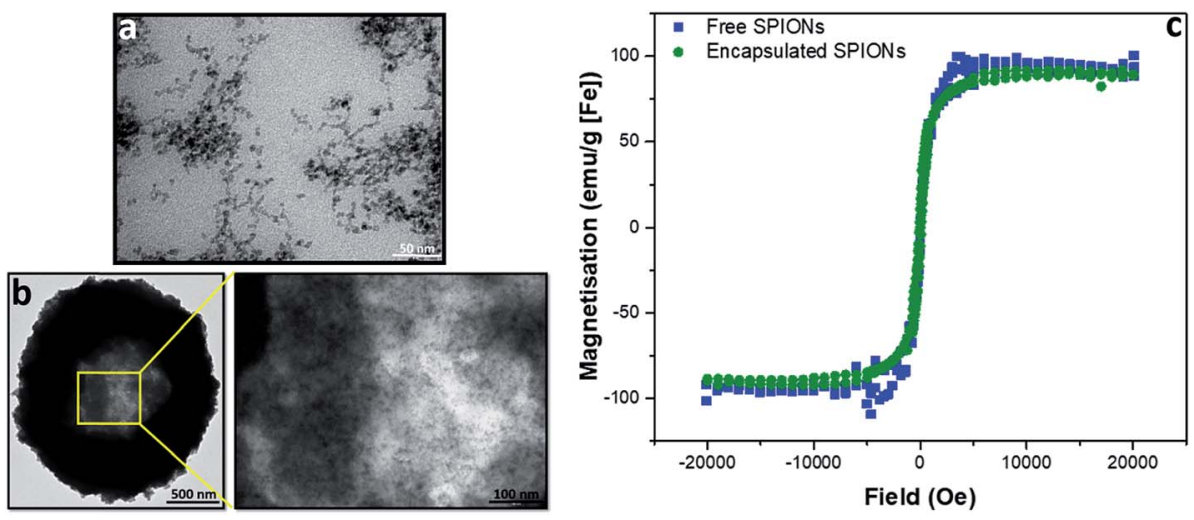

Fig. 1 Characterization of free and encapsulated SPIONs by transmission electron microscopy (TEM) and superconducting quantum interference device (SQUID). TEM of free and encapsulated SPIONs is presented in (a) and (b), respectively. SQUID magnetisation curves are shown in (c). 


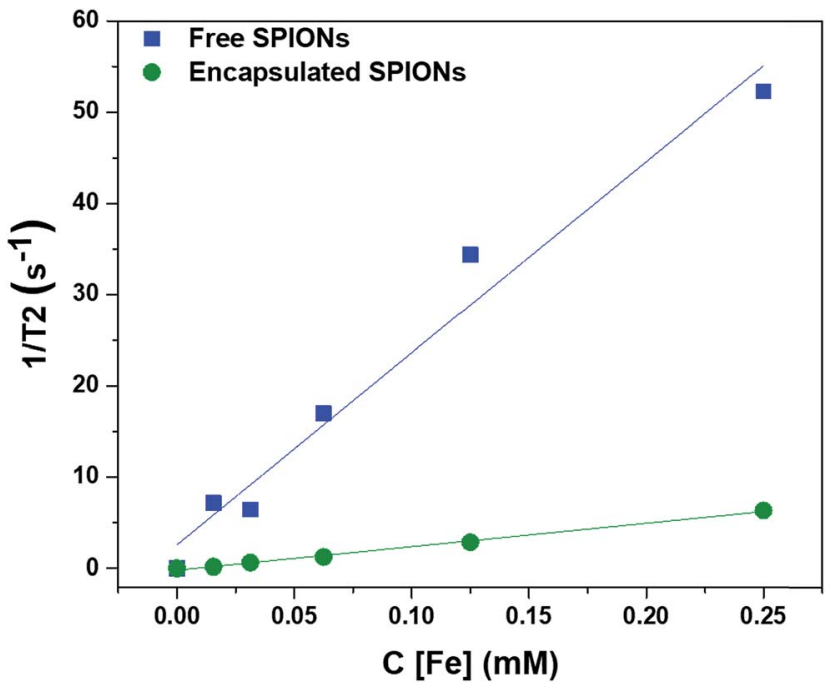

Fig. 2 Effect of encapsulation of SPIONs on the solution relaxivity. The water relaxation rates are plotted against the concentrations of particles (in terms of Fe content).

solution relaxation times (ms; Fig. 3a and b) and the Fe content in labelled cells was determined with a ferrozine assay (Fig. 3c). This revealed a two-fold higher Fe uptake by the cells when labelled with encapsulated SPIONs as compared to the free particles.

The difference in Fe uptake is unlikely to be due to electrostatic interactions, ${ }^{25,33}$ as both formulations were highly positively charged (Fig. SI.1c and $\mathrm{d} \dagger$ ). However, zeta potential measurements for large sedimenting objects (encapsulated SPIONs) is not reliable as sedimentation during the measurement period affects the accuracy of the data. Due to the large number of SPIONs per capsule, the uptake of a few capsules per cell resulted in increased Fe uptake per cell (Fig. 3c). Depending upon the cell line/type and size/dimensions of capsules, the number of micro capsules phagocytosed by cells cannot exceed a limit of 2-15 capsules per cell. ${ }^{33}$ So for low labelling concentrations a higher Fe uptake is achieved by using encapsulated particles. Higher degrees of labelling have been reported with high labelling concentrations of free particles. ${ }^{19}$ In addition to efficient labelling at low Fe concentrations, capsules can be used to combine nanoparticles in a single entity and hence can facilitate multiplexed imaging and measurements. ${ }^{\mathbf{4 3}}$

The toxic effect of free and encapsulated particles is comparable except at high doses of 125 and 250 capsules added per cell (Fig. 3d). This may be due to the sedimentation of the excessive encapsulated particles ( $\mu \mathrm{m}$ dimension; all particles cannot be endocytosed) resulting in the formation of a layer
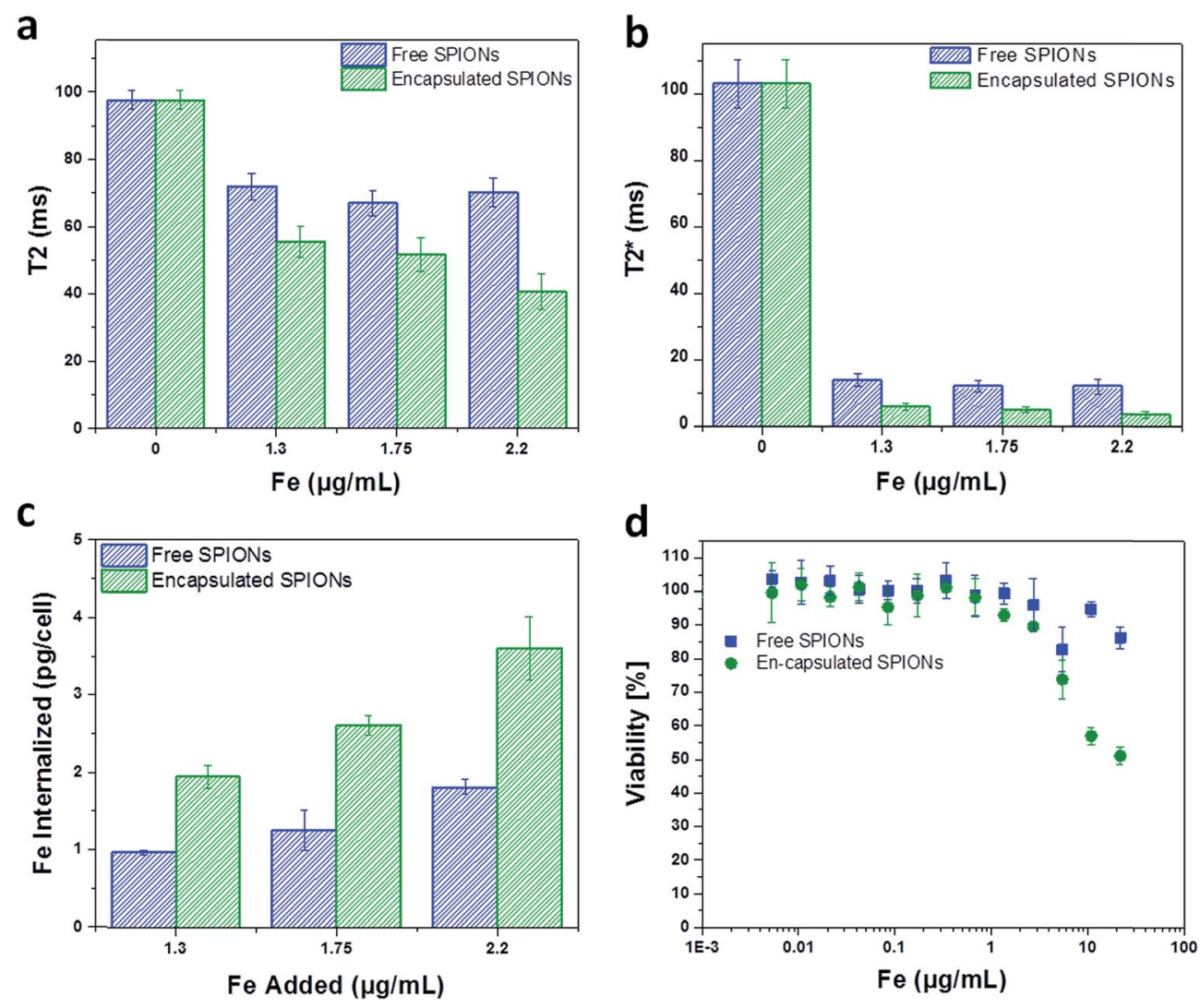

Fig. 3 Cell labelling and toxicity of SPIONs. The solution relaxation times (T2 and T2*) of the particles are plotted against the concentrations used for cell labelling ( $a$ and $b$ ). The labelling concentrations of Fe (Fe added to label the cells) are presented along abcisa. Internalization of free and encapsulated SPIONs versus the added concentration of particles used for cell labelling (determined from ferrozine assay) are shown (c). The Fe concentrations of $1.3,1.75$, and $2.2 \mu \mathrm{g} \mathrm{mL}^{-1}$ corresponds to the doses of 15,20 , and 25 capsules added per cell. Cell viability against the labelling concentrations of $\mathrm{Fe}$ is presented (d). The error bars are the standard deviations of three replicates. 
entirely covering the cells' surface leading to very high local concentration. In the following sections, the cells were labelled with particles below their toxicity level. For in vivo experiments, 15 capsules per cell $\left(1.3 \mu \mathrm{g}\right.$ [Fe] $\left.\mathrm{mL}^{-1}\right)$ and $\sim 2.6$ times higher concentration of free particles $\left(3.3 \mu \mathrm{g}[\mathrm{Fe}] \mathrm{mL}^{-1}\right)$ were added to the cells to obtain a similar amount of iron per cell $(\sim 4$ and $\sim 5$ pg Fe per cell, respectively).

\section{Bioluminescence of mMSCs}

To verify the mMSCs $\mathrm{Luc}^{+}$bioluminescence signal, D-luciferin was added to the cells and the bioluminescence signal was detected after 15 min of incubation using an IVIS spectrum imaging system. It was noticed that the bioluminescence signal from $\sim 200$ cells was detectable (Fig. SI. $2 \dagger$ ), indicating that the intensity of the bioluminescence signal was sufficient for in vivo detection.

\section{Cell phenotype after labelling and re-seeding/re-spreading}

To observe cell phenotype, the trypsinized cells suspended in ice cold PBS for 5-6 h were plated (re-seeded). Three days postseeding the morphology of the labelled cells observed by light microscopy was not distinguishable from unlabeled cells, irrespective of whether they were labelled with free or encapsulated SPIONs (Fig. SI.3†), although more sophisticated morphology analysis after staining could reveal more subtle changes. ${ }^{44}$ The capsules are visible inside the labelled cells by light microscopy (Fig. SI. $3 \dagger$ ). It is well established that after internalization, capsules are localised inside the phago/lysosomes. ${ }^{\mathbf{3 3 4 , 4 5 , 4 6}}$ Homing potential of the mMSCs may be affected by incubation with the particles which can be best checked by staining cell specific markers. ${ }^{47}$

\section{Animal injection and in vivo monitoring of the bio- distribution of labelled cells}

When cells are injected into mice, a significant proportion of them die within a short time-frame..$^{5,42,48}$ Due to the impact on the interpretation of imaging results and particles' associated toxicity, it is important to determine the fate of particles once labelled cells die. Hence, soon after injecting the labelled mMSCs into mice, their in vivo bio-distribution was monitored in parallel by BLI and MRI for 14 days. As expected, the vast majority of the cells were no longer detectable by day 1 . The cells transduced with lentiviral vector $\left(\mathrm{Luc}^{+}\right)$upon interaction with $\mathrm{D}^{-}$ luciferin (injected intraperitoneally in mice) within $15 \mathrm{~min}$ convert D-luciferin to optically active oxyluciferin. The BLI signal of optically active oxyluciferin can be used as indicator of cells viability. ${ }^{\mathbf{1 2}}$ The disappearance of in vivo BLI signal upon injection of D-luciferin confirmed that the majority of the injected cells were dead. Surprisingly, in some animals, a strong luminescence signal was detected in the cardiac regions throughout the course of imaging (Fig. 4); this was likely due to small numbers of cells from the needle tract engrafting in the cardiac muscles following IC administration. By day 14, luminescence signals were present in the hind regions of some mice. As we have previously shown, these were likely to be osteosarcomas resulting from the proliferation of the transplanted cells. ${ }^{11}$ On culling the mice, no tumor was detected in the major body organs (liver, spleen, brain, heart, and lungs). A key objective of this study was to investigate what happened to the free and encapsulated SPIONs after day 1 when BLI showed that the majority of the cells had died. For this purpose, we require consecutive MRI and BLI to be performed on the same animals.

\section{MRI complementary to BLI}

The high resolution of MRI facilitates the visualization of the intra-organ bio-distribution of the contrast agent. ${ }^{11}$ Here, MRI allowed the monitoring of in vivo clearing and accumulation pattern of particles (Fig. 5 and SI.4 $\dagger$ ), after confirmation of cell death by BLI. Since the major Fe accumulation and clearing/ elimination organs are kidneys, liver, and spleen, we imaged the entire abdominal region of mice by MRI throughout the course of experiments to assess the presence and distribution of the particles. Significant difference in contrast can be seen in the images over the time-course of the experiment, being highest soon after injection and reducing towards baseline over the following days (Fig. SI.4†). Full MRI datasets are available on the Zenodo data archive; https://doi.org/10.5281/zenodo.1203991.

To quantify the $\mathrm{Fe}$ accumulation and clearance pattern before and after injection, the $\mathrm{T} 2 *$ relaxation times $(\mathrm{ms})$ in some organs and tissues (liver, spleen, spinal muscles, kidney cortices and medullae) were calculated (Fig. 5) from the MRI scans by drawing regions of interests (ROIs) around these organs and tissues. The most important parameter to track particles by MRI is the generation of negative contrast due to the presence of SPIONs. The decrease in the $\mathrm{T} 2 *$ relaxation times (caused by the dephasing or inhomogeneity from the presence of superparamagnetic iron oxide particles) distorts the local magnetic field, thereby reducing the signal, which appears as dark spots or signal void in MR images. ${ }^{20}$ Hence the presence of more SPIONs (free or encapsulated) resulted in a decrease in the relaxation times generating negative contrast. We have correlated the drop in the relaxation time with the amount of SPIONs present/accumulated inside some organs in vivo. The reversion back of relaxation times close to the baseline values (before injection of labelled cells) gave an indication of clearance of SPIONs from these organs. Soon after injection, the relaxation time decreased in the liver for both free and encapsulated SPIONs and a further decrease can be seen in the following days post-injections. The BLI data indicates that 24 hours postinjection there was considerable cell death. Therefore, the decrease in relaxation time in the liver on day 1 and 2 postinjection is possibly due to SPIONs accumulating in the liver following their release from cells. The presence of SPIONs in the liver up to 48 hours post administration is in accordance to the recent findings of Scarfe et al. ${ }^{11}$ On day 6 post-injection, the relaxation time in the liver started to increase but remained below the baseline value during the entire time course of imaging (Fig. 5). This suggests that the SPIONs accumulated in liver were not completely cleared/eliminated by day 14 . The tendency of accumulation and hence slow clearance of SPIONS from the liver of mice after cell death is similar to the fate of free particles systemically injected in mice. ${ }^{36}$ 

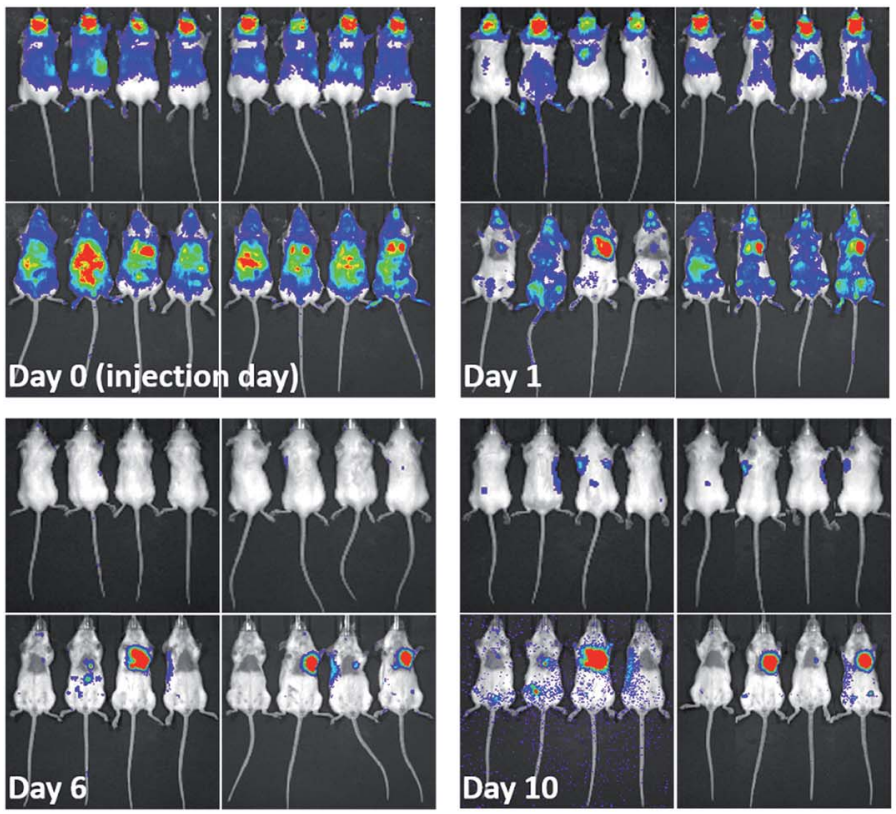
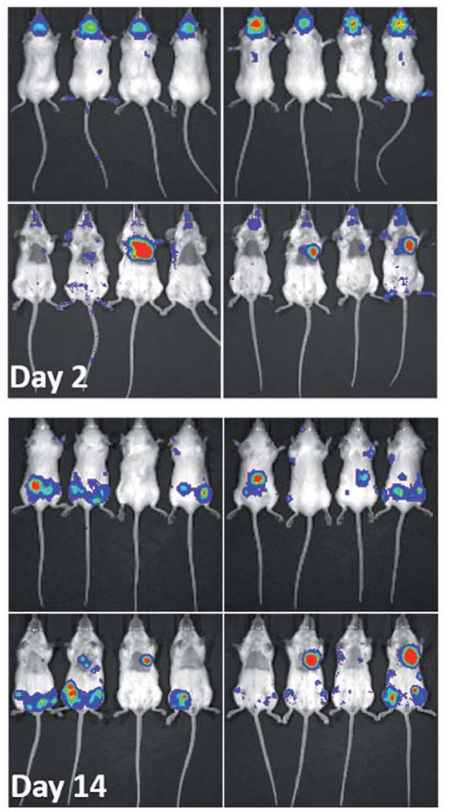

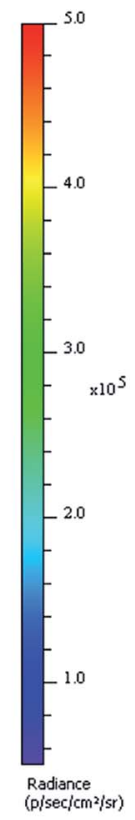

Fig. 4 In vivo long term fate of labelled mMSCs determined from bioluminescence imaging. Images taken at different days post injection of labelled cells. Left panels in each image set (each day) represent mice injected with mMSCs labelled with free SPIONs. While, right panels represent mice injected with mMSCs labelled with encapsulated SPIONs. Upper and lower rows show dorsal and ventral aspects, respectively. Images were recorded 15 minutes after intraperitoneal (IP) injection of D-luciferin.

The spleen has very short relaxation time values at the baseline imaging time point, and no further reduction was observed until the last imaging day in the case of free SPIONs (Fig. 5). By contrast, relaxation times appeared to be lower for encapsulated SPIONs at day 14 post injection. Interestingly, the relaxation times for encapsulated SPIONs had increased in the liver at this time point, suggesting that after leaving the liver, encapsulated particles accumulated inside the spleen. However, detailed biochemical pathways for Fe accumulation in these organs needs to be explored. Recently, the long term in vivo fate
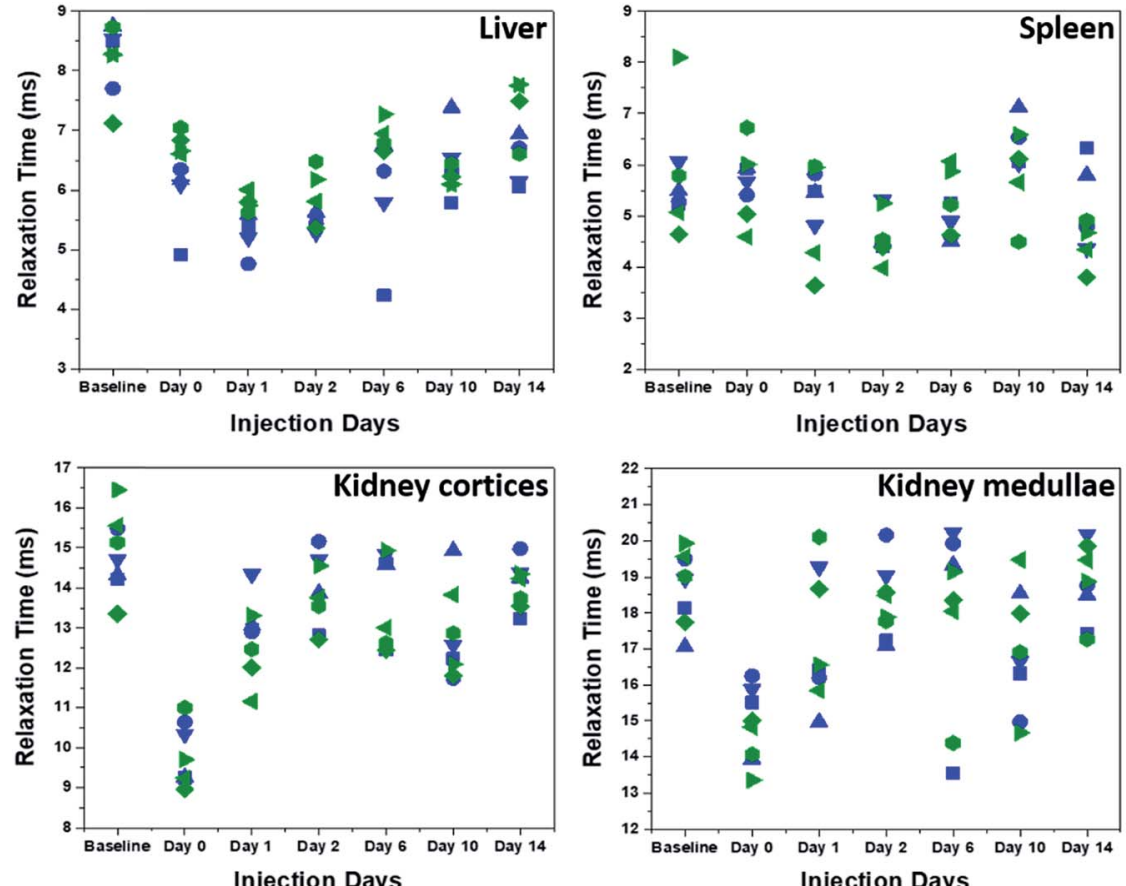

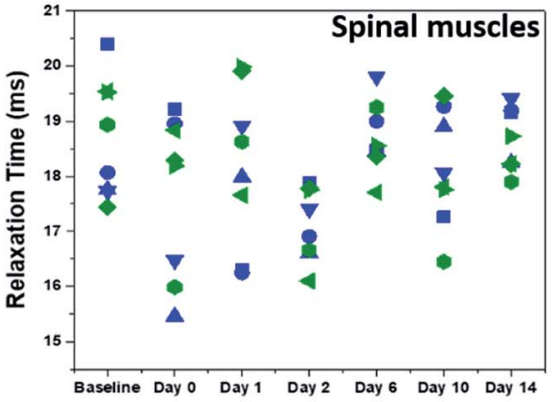

Injection Days

Blue = free SPIONs labelled mMSCs injected mice Green $=$ encapsulated SPIONs labelled mMSCs injected mice Baseline $=$ before injection

Injection Days

Fig. 5 Relaxation times $(\mathrm{T} 2 *)$ as function of time. T2* relaxation times of SPIONs in different organs and tissues are plotted at different days. Different symbol shapes represent different animals. 
of different hybrids of SPIONs with gold nanoparticles has been established after directly injecting the particles in the blood stream of mice which revealed a predominant accumulation of particles in liver and spleen for longer periods of time. ${ }^{36}$ In similar studies, the accumulation and metabolism of particles in the liver and spleen and loss of magnetic characteristics as a function of their surface coating, inner core and formation of protein corona was evaluated. ${ }^{37-40}$ The electron paramagnetic resonance (EPR) based measurements of iron oxide nanocubes showed their considerable elimination from liver on day 7 post administration, whereas half of the proportion of particles stored in the spleen was still detected on day 7 post injection. ${ }^{39,40}$ In general, free SPIONs have faster lysosomal degradation and cellular elimination rate as compared to the aggregated particles. ${ }^{49}$ Here it is important to mention that the SPIONs can be degraded inside the cells before their in vivo release upon cell death. ${ }^{50}$ We speculate that the polymer coating by non-biodegradable materials might reduce the intra-cellular degradation of SPIONs in a similar way that gold coating has been shown to successfully protect the iron oxide core from intra-cellular degradation. ${ }^{51}$

The relaxation time and MRI contrast in spinal muscles did not change considerably throughout the time course of experiments (Fig. 5 and SI.4†). The SPIONs detected in the kidney cortices and medullae immediately after administration were mostly cleared by day 1 post injection (Fig. 5). This finding suggests that after cell death, the particles were rapidly cleared from the kidneys, in line with what has been reported for the intravenous and IC injected particles where particle accumulation inside kidneys is not observed. ${ }^{\mathbf{1 1}}$

\section{Inductively coupled plasma-optical emission spectrometry (ICP-OES) based Fe quantification}

To confirm the results of the MRI analysis, at the end point of imaging experiments the animals were culled and ex vivo ICPOES based Fe quantifications in 6 organs (brain, kidney, liver, lung, spleen \& heart) were performed (Fig. SI.5†). This analysis did not show a significant difference in the percentage of Fe per dry weight of organs at day 14 post injection from the control group of mice which did not receive any injection. This suggests that the majority of the particles released on cell death were cleared by 2 weeks. Although the sensitivity of such measurements is limited as the presence of high quantities of endogenous $\mathrm{Fe}$ and animal-to-animal variability renders their interpretation difficult. More elaborate measurement strategies, e.g. isotope labelling, ${ }^{37,52,53}$ could be employed in future studies.

\section{Materials and methods}

\section{Materials and reagents}

Diethylaminoethyl (DEAE)-dextran ( $\left.M_{\mathrm{w}} 40 \mathrm{kDa}, \# 80881\right)$, iron(III) chloride hexahydrate (\#1.03943), iron(II) chloride tetrahydrate (98\%, \#380024), ammonium hydroxide solution (28-30\%, \#221228), sodium azide (\#S2002), calcium chloride dihydrate (\#223506), sodium carbonate (\#S7795), sodium chloride (\#S7653), poly(sodium 4-styrenesulfonate) (PSS, $M_{\mathrm{w}} \sim 70 \mathrm{kDa}$,
\#243051), dextran (average $M_{\mathrm{w}} 1500-2800 \mathrm{kDa}$, \#D5376), ethylenediaminetetraacetic acid disodium salt dihydrate (EDTA, \#E5134), sterile dimethyl sulfoxide (DMSO, \#D2650), Sephadex® G-100 beads (\#GE17-0060-01), 3-(2-pyridyl)-5,6-di(2furyl)-1,2,4-triazine- $5^{\prime}, 5^{\prime \prime}$-disulfonic acid disodium salt (ferrozine reagent, \#82940), 2-hydroxyethyl agarose (low gelling temperature, \#A4018 and \#A9414), sucrose (\#S7903), L-ascorbic acid (\#95209), ammonium acetate (\#A1542), neocuproine (\#N1501), and Corning®spin-filters (100 kDa, \#CLS431491), were purchased from Sigma Aldrich. Spectra/pore biotech cellulose ester (CE) dialysis membranes (MWCO: $100 \mathrm{kDa}$, \#11415949) and Millex GP syringe filters with polyethersulfone (PES) membranes $(0.22 \mu \mathrm{m}, \# 10038041)$ were purchased from Fisher Scientific, UK. Membrane filters (\#SLHAO33SS; pore size $0.45 \mu \mathrm{m}$, and \#780532; $0.22 \mu \mathrm{m}$ ) were from Millex HA Millipore and STARLAB, respectively. Poly(allylamine hydrochloride) (PAH, $M_{\mathrm{w}} \sim 120-200 \mathrm{kDa}$, \#43092.09) was purchased from VWR. Gelatin capsules (size 4, \#AGG29214) were from Agar Scientific. Double distilled deionized (Millipore Limited, Hertfordshire, UK) water with resistivity of $18.2 \mathrm{M} \Omega \mathrm{cm}$ was used in all experiments.

Luciferase transduced bone marrow derived mouse mesenchymal stem cell line (mMSCs; D1 ORL UVA [D1]ATCC®, \#CRL$12424^{\mathrm{TM}}$ ) was generously provided by Dr Antonius Plagge and was a gift from Bryan Welm (Addgene plasmids \#21375 and 39196). For cell culture, Dulbecco's modified Eagle's medium (DMEM, \#D6546), phosphate buffered saline (PBS; \#806544), PBS tablets (\#P4417), and penicillin-streptomycin (P/S, \#P4333) were purchased from Sigma-Aldrich. L-Glutamine (\#25030081), fetal bovine serum (FBS; \#16000044), and 0.5\% trypsin-EDTA (\#15400054) were purchased from GIBCO (Life Technologies). Paraformaldehyde (PFA, 16\%, \#28908) was from Thermo Fisher Scientific. Cells were counted by an automated cell counter $\left(\mathrm{TC} 20^{\mathrm{TM}}\right)$ from Bio Rad. CellTiter-Glo® reagent (\#G7570) was from Promega. Cell culture Petri dishes (PS, 100/20 mm, vents, Cellstar \#664970), and falcon tubes (Cellstar ${ }^{\circledR} ; 15 \mathrm{~mL}$; \#188271, and $50 \mathrm{~mL}$; \#227261) were from Greiner Bio-one. 24 well plates (\#3524) were from Costar (Corning). 100 well transparent (\#167008) and opaque bottom plates (\#CLS3917) were from Thermo Scientific and Sigma Aldrich, respectively. Eppendorfs (1.5 mL; \#0030120086) were from Eppendorf. 100 (\#981103) and $200 \mu \mathrm{L}$ (\#I1402-8100) polypropylene tubes (Eppendorfs) were purchased from Qiagen and STARLAB, respectively. Centrifuge machines (Sigma 2-6E) and (Heraeus Pico 21, Thermoelectron Corporation) were used for centrifuging the mMSCs. Formvar/carbon-coated 200 mesh copper grids (\#F077/100) were purchased from TAAB.

\section{Characterization tools}

The hydrodynamic diameter and zeta potential measurements were performed in water using a Malvern zetasizer Nano ZS (dynamic light scattering). According to the manufacturer's instructions, the ATP assay (Cell Titer-Glo luminescent assay, Promega) based luminescence (for determination of viability of cells) and ferrozine reagent assay based colorimetric changes (for quantification of iron content) were measured with Fluostar 
Omega (BMG Labtech) coupled with a microplate reader. Transmission electron microscope (TEM) micrographs were taken using a Tecnai G3 spirit TEM at $120 \mathrm{keV} .5 \mu \mathrm{L}$ droplets of the diluted samples were drop-casted onto the formvar/carboncoated 200 mesh copper grids and air dried before TEM analysis. The morphology of mMSCs was observed by bright field (Leica light microscope DM IL coupled with Leica DFC 420C camera) microscopy. For ICP-OES Agilent 5110 ICP-OES spectrometer (with SVDV detection and equipped with the sample changer) was used. To determine the crystalline state and mean core diameter of free SPIONs powder X-ray diffraction (pXRD) measurements were carried out on a Panalytical X'pert Pro multipurpose diffractometer (Co K $\alpha$ source, $\lambda=1.78 \AA$, patterns measurement $>20-120^{\circ} 2 \theta$ for 2 hours, step size $=0.033^{\circ}$, time $/$ step $=295.3 \mathrm{~s}$, scan speed $\left.=0.014^{\circ} \mathrm{s}^{-1}\right)$. Particles samples were freeze-dried using Labconco freezone 4.5 (condenser temperature $-50{ }^{\circ} \mathrm{C}$, shelf temperature $20^{\circ} \mathrm{C}$ ) and their magnetization behaviour (magnetic isotherm) was recorded by means of a superconducting quantum interference device (SQUID) magnetometer (MPMS XL-7, quantum design, USA) at $300 \mathrm{~K}$ with maximum applied field $2 \mathrm{~T}$ under helium atmosphere. For SQUID measurements, freeze-dried samples (a few $\mathrm{mg}$ ) were embedded in gelatin capsules (size 4) which were suspended in the middle of plastic drinking straws. Organs were freeze-dried to obtain their dry weight before ICP-OES based quantification of iron. For bioluminescence (BLI) measurements a bioluminescence imager (IVIS Spectrum, Perkin Elmer, UK) was used to see the in vivo biodistribution of mMSCs. Magnetic resonance imaging (MRI) was performed with Bruker Avance III spectrometer interfaced to a 9.4 T magnet system (Bruker Biospec 90/20 USR) using a $40 \mathrm{~mm}$ transmit/receive volume coil for SPIONs phantoms, SPIONs-labelled cell phantoms and animal imaging. Ultrasound system (Prospect) with ultrasound pulse sequence (UPS) user interface from S-Sharp Corporation Taiwan was used during IC injections of labeled mMSCs to mice.

\section{Animals}

6-8 week old 11 female BALB/c mice were obtained from Charles River, UK and housed in groups of 4 with ad libitum access to standard food and water (ventilated cages, $12 \mathrm{~h}$ light/ dark cycle). Animal experiments were approved by the University of Liverpool (UoL) ethics committee and performed under a licence granted by the UK Home Office under the Animals (Scientific Procedures) Act 1986. Reporting of experiments is in line with the ARRIVE guidelines. Animals were closely observed for any side effects and were culled at the end of experiments (day 14 post injection) without observing a noticeable tumor mass.

\section{Methodology}

Synthesis of super-paramagnetic iron oxide (maghemite) nanoparticles (SPIONs). SPIONs were synthesized following the published protocol from Barrow et al. ${ }^{\mathbf{1 9 , 2 0}}$ Briefly, maghemite (SPIONs) were synthesized by mixing DEAE-dextran (0.05 g, $M_{\mathrm{w}}$ $40 \mathrm{kDa})$, ferric chloride hexahydrate $(0.03 \mathrm{~g})$, and ferrous chloride tetrahydrate $(0.015 \mathrm{~g})$ in $25 \mathrm{~mL}$ water with non-magnetic (polytetrafluoroethylene) stirring under an air-tight connection. The mixture was purged with nitrogen on ice (30 minutes) and ammonium hydroxide ( $1 \mathrm{~mL}, 28-30 \%$ ) was added dropwise (in $2 \mathrm{~min}$ ) under stirring (200 $\pm 5 \mathrm{rpm})$. This was followed by mixture transfer from ice to an oil bath (pre-set at $60{ }^{\circ} \mathrm{C}$ ) and temperature elevation to $80^{\circ} \mathrm{C}$ under nitrogen (in $15 \mathrm{~min}$ ) and then left at $80{ }^{\circ} \mathrm{C}$ for $1 \mathrm{~h}$. Afterwards, the product was brought under air, and refluxed for $5 \mathrm{~h}$ at $110^{\circ} \mathrm{C}$. SPIONs were purified at room temperature by dialysis ( $100 \mathrm{kDa}$ membrane) until the $\mathrm{pH}$ became neutral (7). After concentrating by spin filter (1-2 mL), the sample was passed through size exclusion beads (dextranbased G-100 Sephadex®) to remove excess free polymer. Finally, the particles were washed thrice with deionized water by spin filteration. SPIONs were passed through $0.22 \mu \mathrm{m}$ polyethersulfone (PES) membrane for sterilisation before use in experiments and saved at $4{ }^{\circ} \mathrm{C}$.

Synthesis of SPIONs encapsulating PEM capsules. SPIONs were encapsulated by a modified co-precipitation method, ${ }^{28,34}$ and LbL assembly of oppositely charged layers of polymers and SPIONs around co-precipitated cores. PEM capsules consisting of 32 layers [(PSS/PAH/PSS/SPIONs)7/(PSS/PAH)2] of nonbiodegradable polymers, i.e., PSS and PAH, and SPIONs were fabricated around sacrificial template cores comprising of calcium carbonate incorporating SPIONs and high molecular weight dextran. In order to co-precipitate SPIONs and dextran with calcium carbonate microparticles, solutions of calcium chloride and sodium carbonate were mixed under vigorous stirring in the presence of SPIONs and dextran at room temperature. For this in a glass vial, $4.2 \mathrm{~mL}$ mixture $(0.3 \mathrm{~mL}$ SPIONs; $0.95 \mathrm{mg} \mathrm{mL}^{-1}, 1 \mathrm{~mL}$ dextran; $6.5 \mathrm{mg} \mathrm{mL}^{-1}$, and $2.9 \mathrm{~mL}$ water) was added to $3 \mathrm{~mL}$ of calcium chloride solution $(0.33 \mathrm{M})$. $3 \mathrm{~mL}$ of sodium carbonate solution $(0.33 \mathrm{M})$ was quickly added to the above mixture and continuously stirred for $30 \mathrm{~s}$, under vigorous magnetic stirring (1500 rpm) followed by keeping the reaction contents without agitation for $2 \mathrm{~min}$ at room temperature. The resulting calcium carbonate particles incorporating SPIONs and dextran were washed thrice with deionized water and used for LbL assembly of oppositely charged polyelectrolytes (2 mg mL ${ }^{-1}$ in $\left.0.5 \mathrm{M} \mathrm{NaCl}\right)$ and SPIONs $(0.5 \mathrm{~mL}$ SPIONs; $0.95 \mathrm{mg} \mathrm{mL}^{-1}, 4 \mathrm{~mL}$ water, and $0.5 \mathrm{~mL}$; $0.5 \mathrm{M}$ sodium chloride). The alternating layers of negatively and positively charged polymers, and SPIONs were electrostatically deposited around the charged sacrificial microparticle template cores following an established protocol. ${ }^{54,55}$ For this the microparticles were alternatively immersed inside the solutions of either layer (5 mL; PSS, PAH, or SPIONs), exposed to sonication for short time (1-3 $\mathrm{min}$ ) and kept under agitation/shaking for $13 \mathrm{~min}$. After deposition of each layer the particles were washed thrice with deionized water to remove excess reactants (polymers/SPIONs, etc.). Finally, the cores of PEM capsules were dissolved by complexion of calcium ions with EDTA $(5 \mathrm{~mL}$, $0.2 \mathrm{M}, \mathrm{pH} 6.5 ; 30 \mathrm{~min}$ incubation), washed with deionized water, and stored at $4{ }^{\circ} \mathrm{C}$ after addition of $10.5 \mathrm{~mL}$ water for further use. The capsules diameter was $\sim 2 \mu \mathrm{m}$ as observed from TEM analysis. Further in the manuscript these PEM capsules incorporating SPIONs are called encapsulated SPIONs. 
For SQUID measurements, free and encapsulated SPIONs were freeze-dried and SQUID measurements, TEM, pXRD (free SPIONs), zeta size and potential measurements were performed as mentioned in the characterization section.

Ferrozine assay and ICP-OES based Fe quantification. The samples (particles and particle-labelled cells) were dissolved in 1.2 $\mathrm{M} \mathrm{HCl}$. Fe standard curves were made from the known concentrations of $\mathrm{Fe}$ and from these standard curves the unknown concentrations of $\mathrm{Fe}$ in the samples were determined.

For ferrozine assay, the Fe standard $(50 \mu \mathrm{L}, 4 \mu \mathrm{g}$ in $1.2 \mathrm{M}$ $\mathrm{HCl})$ was serially diluted in $\mathrm{HCl}(1.2 \mathrm{M})$ followed by addition of $50 \mu \mathrm{L}$ water so that final Fe concentration in standards was 2, 1 , 0.5, 0.25, 0.125, 0.0625, $0.031 \mu \mathrm{g}$ in $\mathrm{HCl}(0.6 \mathrm{M} ; 100 \mu \mathrm{L})$. Blank was $100 \mu \mathrm{L} \mathrm{HCl}(0.6 \mathrm{M})$. In the samples with unknown $\mathrm{Fe}$ content, $50 \mu \mathrm{L} \mathrm{HCl} \mathrm{(1.2} \mathrm{M)} \mathrm{was} \mathrm{added} \mathrm{and} \mathrm{samples,} \mathrm{Fe} \mathrm{stan-}$ dards and blank were placed on a heat block at $65{ }^{\circ} \mathrm{C}$ for $2 \mathrm{~h}$. After heat-assisted acid dissolution, the samples and standards were cooled down to room temperature and centrifuged to collect the condensation products along the walls of tubes. $\mathrm{HCl}$ concentration was adjusted to $0.6 \mathrm{M}$ by adding water. $30 \mu \mathrm{L}$ ferrozine reagent was added to $100 \mu \mathrm{L}$ of each sample including standards and blank, mixed and left at room temperature for $30 \mathrm{~min}$. Their absorption was measured at 590 and $780 \mathrm{~nm}$ using a plate reader. For background correction the absorbance at $780 \mathrm{~nm}$ was subtracted from the absorbance at $590 \mathrm{~nm}$ and blank was subtracted from all values. A standard curve for Fe content was plotted and was used to quantify Fe contents in the samples with unknown Fe concentrations.

(Note: the ferrozine reagent was prepared by dissolving $2.4 \mathrm{~g}$ ammonium acetate, $2.2 \mathrm{~g}$ ascorbic acid, $0.02 \mathrm{~g}$ ferrozine, and $0.02 \mathrm{~g}$ neocuproine (dissolved in small volume of ethanol) in $6.25 \mathrm{~mL}$ water, mixed, frozen in small aliquots and protected from light).

For ICP-OES Fe quantifications after dissolution with $1.2 \mathrm{M}$ $\mathrm{HCl}$, the samples were diluted in water so that final acid content will not exceed 5\%. ICP-OES measurements were performed using 3 readings per wavelength and 11 wavelengths per sample. The concentration determination was performed using calibration curve for Fe consisting of 6 measurement points of freshly prepared Fe concentrations $\left(0-10 \mu \mathrm{g} \mathrm{mL}{ }^{-1}\right)$ derived from iron standard solutions from Inorganic Ventures $\left(100 \mathrm{mg} \mathrm{L}^{-1}\right)$.

Cell viability measurements. CellTiter-Glo® reagent was used for cell viability measurements. For this assay, mMSCs were seeded in 96 well transparent bottomed plates at 10000 cells per well in $100 \mu \mathrm{L}$ complete cell growth medium (DMEM supplemented with $10 \% \mathrm{FBS}, 1 \% \mathrm{P} / \mathrm{S}$, and $1 \%$ L-glutamine). After $24 \mathrm{~h}$ cells were incubated with free and encapsulated SPIONs suspended in fresh cell culture medium for $24 \mathrm{~h}$. Serial dilution of particles was performed in complete cell growth medium. Each condition was applied in triplicate. After incubation with particles, the cells were washed with PBS and $100 \mu \mathrm{L}$ fresh cell growth medium was added to each well of assay plates, followed by the addition of $20 \mu \mathrm{L}$ CellTiter-Glo® reagent. The plates were shaken at 600-700 rpm in an orbital shaker for 1 min (to complete cell lysis), incubated at room temperature for $5 \mathrm{~min}$ and $100 \mu \mathrm{L}$ mixture contents from each well of the plates were transfered in opaque bottomed 96 well plates. The luminescence from each well of 96 well plate was measured by a Fluostar Omega (BMG Labtech) plate reader. Viability was expressed as \% of the untreated control.

Phantoms for MR measurements. Phantoms for MR measurements were prepared in 1\% low melting agarose.

1 Phantoms of particles. Low melting agarose was dissolved in water at $65{ }^{\circ} \mathrm{C}$ to get a clear $2 \%$ solution and placed at $40{ }^{\circ} \mathrm{C}$ during mixing and addition of particles. The solutions of SPIONs (free and encapsulated) were diluted in water (serial dilution) to get $0.5,0.25,0.125,0.0625$, and $0.03125 \mathrm{mM} \mathrm{Fe}$ concentrations. These diluted solutions of particles were mixed with the gel at $40{ }^{\circ} \mathrm{C}(1: 1$ dilution by volume; $100 \mu \mathrm{L}$ of each sample was mixed throughly with $100 \mu \mathrm{L}$ gel without bubble formation in $200 \mu \mathrm{L}$ polypropylene tubes) to get final Fe content $0.25,0.125,0.0625,0.03125$, and $0.0156 \mathrm{mM}$ in $1 \%$ agarose. $2 \%$ agarose was mixed with water $(1: 1$ dilution) to serve as control. These samples were mounted in $1 \%$ agarose containing holders, stored at $4{ }^{\circ} \mathrm{C}$ for $24 \mathrm{~h}$ and analyzed by MRI. The MR measurement parameters are listed in Table SI.1. $\dagger$ The solution relaxivity of the particles entrapped in phantoms was calculated from their relaxation times which were acquired by multi gradient echo sequences (MGES) and the imaging was performed with a fast low angle shot (FLASH) sequence.

2 Phantoms of labelled cells. In 6 well plates mMSCs were seeded $\left(2 \times 10^{5}\right.$ cells per well $)$ in $2 \mathrm{~mL}$ complete growth media. After $24 \mathrm{~h}$ the free and encapsulated SPIONs were added at 15, 20 , and 25 capsules per cell (equivalent to a dose of 13.1, 17.5, and $21.8 \mathrm{pg}$ Fe in free SPIONs per cell) considering the seeded number of cells. After $24 \mathrm{~h}$ of incubation, cells were washed twice with PBS and trypsinized. Particle-labelled cells were centrifuged at $500 \mathrm{rcf}$ for $5 \mathrm{~min}$ and the supernatant was removed. The cell pellets were resuspended in 4\% PFA, mixed without bubble formation and fixed cells were transfered in small polypropylene tubes $(100 \mu \mathrm{L}$ for each condition) centrifuged and maximum supernatant was removed. The cell pellets were resuspended in PBS and mixed with $2 \%$ low melting agarose (1:1 dilution). $3 \times 10^{5}$ fixed cells were poured on the top of already solidified $1 \%$ agarose gel. The samples were mounted in 1\% agarose containing holders and imaged with a fast low angle shot (FLASH) sequence. 19 images per sample were captured. Their $\mathrm{T} 2$ and $\mathrm{T} 2 *$ relaxation times were measured with MGES. Imaging parameters are listed in Table SI. $2 . \dagger$

For ferrozine based Fe quantification, the particles labelled cells (after washing and trypsinization) were counted, centrifuged and dissolved in $\mathrm{HCl}(1.2 \mathrm{M})$ after removing maximum supernatant.

Luc $^{+}$activity of mMSCs. The cells were monitored for $\mathrm{Luc}^{+}$ activity before in vivo experiments. For this mMSCs were seeded in 96 well plates in triplicate with serial dilutions $(10000,5000$, $2500,1250,625,312,156,78,39,20,7,3,2,1$, and 0 cells per well) in $100 \mu \mathrm{L}$ complete growth media and cultured at constant (5\%) supply of $\mathrm{CO}_{2}$ for $24 \mathrm{~h}$ at $37^{\circ} \mathrm{C}$. After refreshing the growth media, the plates were placed in IVIS spectrum imaging system, and noted the baseline bio-luminescence signals. Latter, their growth media was replaced by fresh growth media $(100 \mu \mathrm{L})$

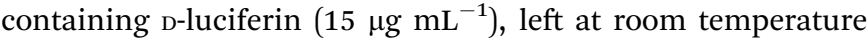


for $15 \mathrm{~min}$ and their bio-luminescence was recorded and expressed as radiance (photons per second per $\mathrm{cm}^{2}$ per steradian $\left.\left[\mathrm{p} \mathrm{s}^{-1} \mathrm{~cm}^{-2} \mathrm{sr}^{-1}\right]\right)$.

Cell labelling and administration in mice. mMSCs transduced with lentiviral vector $\left(\mathrm{Luc}^{+}\right)$were IC injected in mice. The vector details are described in a recent study. ${ }^{12}$ To label cells with particles, $1.5 \times 10^{6} \mathrm{mMSCs}$ were seeded in $100 \mathrm{~mm}$ tissue culture dishes and incubated for $24 \mathrm{~h}$ at $37{ }^{\circ} \mathrm{C}$ in humidified atmosphere with $5 \% \mathrm{CO}_{2}$. Free $(33.72 \mathrm{pg} \mathrm{Fe}$ per cell) and encapsulated (@15 capsules per cell equivalent to a dose of 13.1 pg Fe per cell) SPIONs were added to the cells considering the seeded number of cells and incubated for $24 \mathrm{~h}$. The cells were washed twice with PBS, trypsinized, kept on ice (after removing trypsin), and resuspended in ice cold PBS after cell counting. To observe the morphology of cells after particles labelling, some of the trypsinized cells were re-seeded and imaged after 3 days. $10^{6}$ cells in $100 \mu \mathrm{L}$ PBS (having $\sim 5$ and $\sim 4 \mathrm{pg}$ Fe per cell for free and encapsulated SPIONs, respectively) were IC injected into the left ventricles of female $\mathrm{BALB} / \mathrm{c}$ mice under ultrasound guided injection. Details of IC injections is provided in a recent study. ${ }^{11}$ Briefly, mice were anesthetized with a mixture of isoflurane and oxygen, subcutaneous (SC) injected with an analgesic buprenorphine ( $0.1 \mathrm{mg} \mathrm{kg} \mathrm{kg}^{-1}$ body weight) and were positioned supine above a heated platform. Their fur was removed and the limbs and abdomen were taped after extension of body (to hold body in extended position and the skin over the chest taut). Ultrasound gel was applied to the chest, and ultrasound transducer was positioned over the mice to have chest in view. Visualized the heart and labelled cells were injected inside the left cardiac ventricle with the help of 29G 1/2 inch insulin syringe. The cells were completely re-suspended, and were injected in a slow and well controlled manner. Soon after IC injections mice were imaged by MR under the same anaesthesia session and latter in groups of 4 by IVIS based BLI, on 0 (injections day), 1, 2, 6, 10, and 14 days post injections. Where possible mice were imaged by both imaging modalities under same anesthetic session. The mice were recovered from anaesthesia in a heat box set at $32{ }^{\circ} \mathrm{C}$, and closely monitored for the signs of any adverse effects during the course of studies.

In vivo MR imaging. The biodistribution of cells in the abdominal region, i.e., kidneys, liver, spinal muscles, and spleen was imaged with a 9.4 T horizontal bore MRI scanner (Bruker Avance III spectrometer) using a $40 \mathrm{~mm}$ transmit/ receive volume coil. FLASH $\mathrm{T}_{2} *$ weighted sequences were recorded and $\mathrm{T} 2 *$ maps were generated using MGES. A prescan before injections served as control to set baseline value for MR analysis. Regions of interests (ROIs) were drawn around the kidney cortices, medullae, liver, spleen and spinal muscles and $\mathrm{T} 2 *$ relaxation times were calculated from the $\mathrm{T} 2 *$ maps. MR acquisition parameters are listed in Table SI.3. $\dagger$

Bioluminescence imaging (BLI). BLI complementary to each MR scan was performed until the last imaging day (day 14 post injection). Mice received D-luciferin (150 $\mathrm{mg} \mathrm{kg}^{-1}$ body weight) intraperitoneally and were imaged $15 \mathrm{~min}$ post injections by IVIS spectrum imaging system. Imaging was performed by 13 min luminescence exposure and expressed as radiance (photons per second per $\mathrm{cm}^{2}$ per steradian $\left[\mathrm{p} \mathrm{s}^{-1} \mathrm{~cm}^{-2} \mathrm{sr}^{-1}\right]$ ).
ICP-OES based Fe quantification in mice organs. For quantification of $\mathrm{Fe}$ content in the brain, kidneys, liver, lungs, spleen, and heart, at the end of in vivo experiments mice were euthanized under terminal anesthesia followed by cervical dislocation. After dissection, the above mentioned organs were collected, washed with PBS and preserved in 70\% ethanol. Organs collected from 3 untreated mice served as control. The organs in $70 \%$ ethanol were dipped in $96 \%$ ethanol, crushed with pestle and mortar and left overnight for freeze-drying. The dried crushed organs mass was weighed. $0.5 \mathrm{~mL} \mathrm{HCl}(1.2 \mathrm{M})$ was added to the dried and crushed organs, kept inside an oven at $70{ }^{\circ} \mathrm{C}$ for $3 \mathrm{~h}$ and brought the reaction contents at room temperature. Water was added to each sample vial to get $10 \mathrm{~mL}$ final volume. Samples were filtered through $0.2 \mu \mathrm{m}$ filters to remove organ debris. Fe content in each sample was determined by ICP-OES. The dried weight of crushed organs noted before acid digestion was used for normalizing Fe content per dried weight of each organ.

\section{Conclusions}

We have demonstrated that SPIONs can be encapsulated inside polymeric capsules while retaining their individual particle identities and demonstrated similar magnetization behavior as free particles. Although the solution relaxivity of free particles was very different from encapsulated ones, once internalized inside lysosomal compartments after cell uptake, this difference became negligible for similar concentrations (Fe) of particles. The encapsulation of particles has some potential advantages over free particles; e.g., (1) in case of cell labelling with small amount of particles, a higher uptake can be achieved, and (2) the addition of different types of particles in a single entity for multiplexed measurements is possible. Comparison of both formulations of SPIONs did not reveal any difference in the in vivo fate of particles upon cell death and was similar to what has been reported for particles directly injected in the blood stream of mice.

\section{Conflicts of interest}

There are no conflicts to declare.

\section{Acknowledgements}

We thank Dr Antonius Plagge for providing Luc ${ }^{+}$mMSCs. The Electron Microscopy Unit, Biomedical Services Unit, and the Centre for Preclinical Imaging at the University of Liverpool are acknowledged for help and support. We are grateful for the support from BBSRC, EPSRC, and MRC-funded UK Regenerative Medicine Platform "Safety and Efficacy, focusing on Imaging Technologies Hub" (MR/K026739/1). This project has received funding from the European Union's Horizon 2020 research and innovation programme under the Marie Skłodowska-Curie grant agreement No. 705600 (MultimodalCellTrack; Fellowship to SA). Pranab Mandal and Stephen Moss are acknowledged for SQUID and ICP-OES measurements, respectively. 


\section{References}

1 A. I. Caplan, J. Cell. Physiol., 2007, 213, 341-347.

2 H. Mizuno, M. Tobita and A. C. Uysal, Stem Cells, 2012, 30, 804-810.

3 S. M. Cromer Berman, P. Walczak and J. W. Bulte, Wiley Interdiscip. Rev.: Nanomed. Nanobiotechnol., 2011, 3, 343-355.

4 E. Buzhor, L. Leshansky, J. Blumenthal, H. Barash, D. Warshawsky, Y. Mazor and R. Shtrichman, Regener. Med., 2014, 9, 649-672.

5 R. Guzman, N. Uchida, T. M. Bliss, D. He, K. K. Christopherson, D. Stellwagen, A. Capela, J. Greve, R. C. Malenka and M. E. Moseley, Proc. Natl. Acad. Sci. U. S. A., 2007, 104, 10211-10216.

6 M. M. Daadi, Z. Li, A. Arac, B. A. Grueter, M. Sofilos, R. C. Malenka, J. C. Wu and G. K. Steinberg, Mol. Ther., 2009, 17, 1282-1291.

7 I. J. M. de Vries, W. J. Lesterhuis, J. O. Barentsz, P. Verdijk, J. H. van Krieken, O. C. Boerman, W. J. Oyen, J. J. Bonenkamp, J. B. Boezeman and G. J. Adema, Nat. Biotechnol., 2005, 23, 1407-1413.

8 A. Taylor, K. M. Wilson, P. Murray, D. G. Fernig and R. Lévy, Chem. Soc. Rev., 2012, 41, 2707-2717.

9 M. F. Kircher, S. S. Gambhir and J. Grimm, Nat. Rev. Clin. Oncol., 2011, 8, 677-688.

10 R. Meir, K. Shamalov, O. Betzer, M. Motiei, M. HorovitzFried, R. Yehuda, A. Popovtzer, R. Popovtzer and C. J. Cohen, ACS Nano, 2015, 9, 6363-6372.

11 L. Scarfe, A. Taylor, J. Sharkey, R. Harwood, M. Barrow, J. Comenge, L. Beeken, C. Astley, I. Santeramo, C. Hutchinson, L. Ressel, J. Smythe, E. Austin, R. Levy, M. J. Rosseinsky, D. J. Adams, H. Poptani, B. K. Park, P. Murray and B. Wilm, bioRxiv, 2017, DOI: 10.1101/202101.

12 J. Comenge, J. Sharkey, O. Fragueiro, B. Wilm, M. Brust, P. Murray, R. Levy and A. Plagge, eLife, 2018, 7, e33140.

13 M. S. Evans, J. P. Chaurette, S. T. Adams Jr, G. R. Reddy, M. A. Paley, N. Aronin, J. A. Prescher and S. C. Miller, Nat. Methods, 2014, 11, 393-395.

14 L. Mezzanotte, M. Aswendt, A. Tennstaedt, R. Hoeben, M. Hoehn and C. Löwik, Contrast Media Mol. Imaging, 2013, 8, 505-513.

15 L. Scarfe, N. Brillant, J. D. Kumar, N. Ali, A. Alrumayh, M. Amali, S. Barbellion, V. Jones, M. Niemeijer and S. Potdevin, NPJ Regen. Med., 2017, 2, 28-40.

16 M. Kooi, V. Cappendijk, K. Cleutjens, A. Kessels, P. Kitslaar, M. Borgers, P. Frederik, M. Daemen and J. Van Engelshoven, Circulation, 2003, 107, 2453-2458.

17 B. Bonnemain, J. Drug Targeting, 1998, 6, 167-174.

18 A. S. Merbach, L. Helm and E. Toth, The chemistry of contrast agents in medical magnetic resonance imaging, John Wiley \& Sons, 2013.

19 M. Barrow, A. Taylor, A. M. Fuentes-Caparrós, J. Sharkey, L. M. Daniels, P. Mandal, B. K. Park, P. Murray, M. J. Rosseinsky and D. J. Adams, Biomater. Sci., 2018, 6, 101-106.
20 M. Barrow, A. Taylor, D. J. Nieves, L. K. Bogart, P. Mandal, C. M. Collins, L. R. Moore, J. J. Chalmers, R. Lévy and S. R. Williams, Biomater. Sci., 2015, 3, 608-616.

21 M. Barrow, A. Taylor, P. Murray, M. J. Rosseinsky and D. J. Adams, Chem. Soc. Rev., 2015, 44, 6733-6748.

22 M. Barrow, A. Taylor, J. García Carrión, P. Mandal, B. K. Park, H. Poptani, P. Murray, M. J. Rosseinsky and D. J. Adams, Contrast Media Mol. Imaging, 2016, 11, 362-370.

23 M. R. Santoso and P. C. Yang, Stem Cells Int., 2016, 2016, 1-9. 24 R. Hachani, M. A. Birchall, M. W. Lowdell, G. Kasparis, L. D. Tung, B. B. Manshian, S. J. Soenen, W. Gsell, U. Himmelreich and C. A. Gharagouzloo, Sci. Rep., 2017, 7, 7850-7863.

25 S. Ashraf, J. Park, M. A. Bichelberger, K. Kantner, R. Hartmann, P. Maffre, A. H. Said, N. Feliu, J. Lee and D. Lee, Nanoscale, 2016, 8, 17794-17800.

26 A. Taylor, A. Herrmann, D. Moss, V. Sée, K. Davies, S. R. Williams and P. Murray, PLoS One, 2014, 9, e100259.

27 C. Billotey, C. Wilhelm, M. Devaud, J. C. Bacri, J. Bittoun and F. Gazeau, Magn. Reson. Med., 2003, 49, 646-654.

28 S. De Koker, R. Hoogenboom and B. G. De Geest, Chem. Soc. Rev., 2012, 41, 2867-2884.

29 L. L. del_Mercato, P. Rivera-Gil, A. Z. Abbasi, M. Ochs, C. Ganas, I. Zins, C. Sönnichsen and W. J. Parak, Nanoscale, 2010, 2, 458-467.

30 P. Rivera Gil, L. L. del Mercato, P. del Pino, A. Munoz Javier and W. J. Parak, Nano Today, 2008, 3, 12-21.

31 L. L. del_Mercato, A. Z. Abbasi and W. J. Parak, Small, 2011, 7, 351-363.

32 A. Z. Abbasi, L. Gutiérrez, L. L. del Mercato, F. Herranz, O. Chubykalo-Fesenko, S. Veintemillas-Verdaguer, W. J. Parak, M. P. Morales, J. M. González and A. Hernando, J. Phys. Chem. C, 2011, 115, 6257-6264.

33 L. Kastl, D. Sasse, V. Wulf, R. Hartmann, J. Mircheski,

C. Ranke, S. Carregal-Romero, J. A. Martínez-López,

R. Fernández-Chacón, W. J. Parak, H.-P. Elsasser and P. Rivera_Gil, ACS Nano, 2013, 7, 6605-6618.

34 P. Rivera_Gil, M. Nazarenus, S. Ashraf and W. J. Parak, Small, 2012, 8, 943-948.

35 J. Sharkey, L. Scarfe, I. Santeramo, M. Garcia-Finana, B. K. Park, H. Poptani, B. Wilm, A. Taylor and P. Murray, Eur. J. Pharmacol., 2016, 790, 74-82.

36 J. Kolosnjaj-Tabi, Y. Javed, L. Lartigue, J. Volatron, D. Elgrabli, I. Marangon, G. Pugliese, B. Caron, A. Figuerola and N. Luciani, ACS Nano, 2015, 9, 7925-7939. 37 N. Feliu, D. Docter, M. Heine, P. del Pino, S. Ashraf, J. Kolosnjaj-Tabi, P. Macchiarini, P. Nielsen, D. Alloyeau and F. Gazeau, Chem. Soc. Rev., 2016, 45, 2440-2457.

38 J. Kolosnjaj-Tabi, J. Volatron and F. Gazeau, Design and Applications of Nanoparticles in Biomedical Imaging, Springer, 2017, pp. 9-41.

39 M. Levy, N. Luciani, D. Alloyeau, D. Elgrabli, V. Deveaux, C. Pechoux, S. Chat, G. Wang, N. Vats and F. Gendron, Biomaterials, 2011, 32, 3988-3999.

40 J. Kolosnjaj-Tabi, L. Lartigue, Y. Javed, N. Luciani, T. Pellegrino, C. Wilhelm, D. Alloyeau and F. Gazeau, Nano Today, 2016, 11, 280-284. 
41 M. M. Lalu, L. McIntyre, C. Pugliese, D. Fergusson, B. W. Winston, J. C. Marshall, J. Granton and D. J. Stewart, PLoS One, 2012, 7, e47559.

42 N. M. Toyserkani, M. G. Jørgensen, S. Tabatabaeifar, C. H. Jensen, S. P. Sheikh and J. A. Sørensen, Stem Cells Transl. Med., 2017, 6, 1786-1794.

43 L. L. del Mercato, A. Z. Abbasi, M. Ochs and W. J. Parak, ACS Nano, 2011, 5, 9668-9674.

44 X. Ma, R. Hartmann, D. Jimenez de Aberasturi, F. Yang, S. J. Soenen, B. B. Manshian, J. Franz, D. Valdeperez, B. Pelaz, N. Feliu, N. Hampp, C. Riethmüller, H. Vieker, N. Frese, A. Gölzhäuser, M. Simonich, R. L. Tanguay, X.-J. Liang and W. J. Parak, ACS Nano, 2017, 11, 7807-7820. 45 A. Ott, X. Yu, R. Hartmann, J. Rejman, A. Schütz, M. Ochs, W. J. Parak and S. Carregal-Romero, Chem. Mater., 2015, 27, 1929-1942.

46 A. Muñoz_Javier, O. Kreft, M. Semmling, S. Kempter, A. G. Skirtach, O. Bruns, P. d. Pino, M. F. Bedard, J. Rädler, J. Käs, C. Plank, G. Sukhorukov and W. J. Parak, Adv. Mater., 2008, 20, 4281-4287.

47 P. Nold, R. Hartmann, N. Feliu, K. Kantner, M. Gamal, B. Pelaz, J. Hühn, X. Sun, P. Jungebluth, P. del Pino, H. Hackstein, P. Macchiarini, W. J. Parak and C. Brendel, J. Nanobiotechnol., 2017, 15, 24.
48 J. Leibacher and R. Henschler, Stem Cell Res. Ther., 2016, 7, 7-18.

49 L. Lartigue, D. Alloyeau, J. Kolosnjaj-Tabi, Y. Javed, P. Guardia, A. Riedinger, C. Péchoux, T. Pellegrino, C. Wilhelm and F. Gazeau, ACS Nano, 2013, 7, 3939-3952.

50 F. Mazuel, A. Espinosa, N. Luciani, M. Reffay, R. Le Borgne, L. Motte, K. Desboeufs, A. Michel, T. Pellegrino, Y. Lalatonne and C. Wilhelm, ACS Nano, 2016, 10, 7627-7638.

51 F. Mazuel, A. Espinosa, G. Radtke, M. Bugnet, S. Neveu, Y. Lalatonne, G. A. Botton, A. Abou-Hassan and C. Wilhelm, Adv. Funct. Mater., 2017, 27, 1605997.

52 D. Bargheer, J. Nielsen, G. Gébel, M. Heine, S. C. Salmen, R. Stauber, H. Weller, J. Heeren and P. Nielsen, Beilstein J. Nanotechnol., 2015, 6, 36-46.

53 A. Carambia, B. Freund, D. Schwinge, O. T. Bruns, S. C. Salmen, H. Ittrich, R. Reimer, M. Heine, S. Huber and C. Waurisch, J. Hepatol., 2015, 62, 1349-1356.

54 S. De Koker, B. G. De Geest, C. Cuvelier, L. Ferdinande, W. Deckers, W. E. Hennink, S. De Smedt and N. Mertens, Adv. Funct. Mater., 2007, 17, 3754-3763.

55 S. Carregal-Romero, M. Ochs, P. Rivera-Gil, C. Ganas, A. M. Pavlov, G. B. Sukhorukov and W. J. Parak, J. Controlled Release, 2012, 159, 120-127. 